\title{
String Perturbation Theory on the Schwinger-Keldysh Time Contour
}

\author{
Petr Hořava $\odot$ and Christopher J. Mogni \\ Berkeley Center for Theoretical Physics and Department of Physics, University of California, \\ Berkeley, California 94720-7300, USA \\ and Lawrence Berkeley National Laboratory, Physics Division, Berkeley, California 94720-8162, USA
}

(Received 15 September 2020; accepted 23 November 2020; published 22 December 2020)

\begin{abstract}
We perform the large- $N$ expansion in the Schwinger-Keldysh formulation of nonequilibrium quantum systems with matrix degrees of freedom and study universal features of the anticipated dual string theory. We find a rich refinement of the topological genus expansion: In the original formulation, the future time instant where the forward and backward branches of the Schwinger-Keldysh time contour meet is associated with its own world sheet genus expansion. After the Keldysh rotation, the world sheets decompose into a classical and quantum part.
\end{abstract}

DOI: 10.1103/PhysRevLett.125.261602

Introduction.-Nonequilibrium many-body systems are of central interest in remarkably many areas of physics, across a vast range of scales: from the microscopic scales of particle physics, to mesoscopic phenomena and condensed matter physics, to the cosmological scales of the cosmic microwave background, and the large-scale structure of the Universe. Moreover, the fluctuations governing the collective behavior in such systems may be either quantum or classical, thermal in nature.

In the past few decades, the paradigm of string theory has proven to be a powerful generator of novel theoretical concepts that have found their way into remarkably many areas of physics and mathematics, not only to quantum gravity and particle phenomenology beyond the standard model, but also to condensed matter in holographic dualities and AdS/CFT correspondence [1,2] or in helping with the topological classification of new topological states and phases of matter [3]. One naturally wonders if this useful influence of string theory could be extended to nonequilibrium systems.

A direct attempt to formulate string theory far away from equilibrium faces a strong, historically rooted obstacle: String theory originated [4] from the theory of the $S$ matrix, which is itself strongly based on the assumption of the static, stable, eternal relativistic vacuum. While a long list of brilliant results have been accumulated for strings out of equilibrium using traditional methods (in particular, in string cosmology), they have been achieved in spite of this obstacle. The purpose of this Letter is to propose steps

Published by the American Physical Society under the terms of the Creative Commons Attribution 4.0 International license. Further distribution of this work must maintain attribution to the author(s) and the published article's title, journal citation, and DOI. Funded by SCOAP. toward eliminating this obstacle from first principles. Having access to a broader formulation of string theory away from equilibrium would benefit a number of subdisciplines, from early Universe cosmology, the physics of black holes, and the information puzzle, to less traditional areas for applied string theory, such as nonequilibrium mesoscopic physics. A duality to string theory may provide access to a new weakly coupled perturbative technique for otherwise strongly correlated nonequilibrium systems, similar to what we witnessed in the context of AdS/CMT correspondence [1,2].

Generally, in many-body physics such an assumption is far from necessary. The more general formulation, which could simply be called "quantum mechanics without simplifying assumptions" about the vacuum, is known as the Schwinger-Keldysh (SK) formalism [5,6] (see [7] for a comprehensive list of reviews). The system is evolved forward and then backward, along a doubled time contour called the SK time contour. Equivalently, this doubling can be viewed as a doubling of fields on the single-valued time $t$. This formalism has been the leading go-to technique for handling nonequilibrium many-body systems in condensed matter and a broad range of related areas for many decades. It also plays an increasingly important role in gravity and cosmology, which goes back to the early pioneering and insightful work by Hájíček [8] and later by Jordan [9]. In this century, the importance of the SK "in-in" formalism for inflationary cosmology has been particularly stressed by Weinberg [10-12] (see also [13]).

Understanding how string theory relates to the SK formalism is an important step toward developing nonequilibrium string theory. Some work on SK formalism and strings already exists in the literature, primarily from the spacetime point of view [14-19]. Here we follow a different strategy: In equilibrium, the structure of the large- $N$ expansion in theories with interacting matrix degrees of 
freedom predicts a string coupling expansion, as a sum over connected world sheet topologies $\Sigma$ of increasing genus,

$$
\mathcal{Z}=\sum_{h=0}^{\infty}\left(\frac{1}{N}\right)^{2 h-2} \mathcal{F}_{h}(\lambda, \ldots) .
$$

Here $g_{s} \equiv 1 / N$ plays the role of the string coupling constant, with the power of $N$ given by the Euler number $\chi(\Sigma)=2-2 h$. The " $t$ Hooft coupling $\lambda$ is a world sheet coupling analogous to $\alpha^{\prime}$ of critical strings, with “..." suggesting there might be more than one such world sheet coupling. The importance of this duality, first developed by 't Hooft [20,21], was further advocated over the years by Polyakov (see, e.g., [22]) and others. It turned out that, at least in special circumstances, when combined with additional spacetime symmetries, this relationship reveals a lot about the dual string theory, eventually leading to such milestones as AdS/CFT correspondence [23]. Can this relationship be extended away from equilibrium, and if so, what does it reveal about the perturbative expansion of the dual string theory?

The advantage of asking this question first on the large- $N$ side is that we understand conceptually quite well how to take that system out of equilibrium: simply apply the SK formalism. On the string dual side, much less is known about nonequilibrium, and we can hope to learn something new by taking the correspondence seriously.

We started this exploration of the large- $N$ expansion in nonequilibrium in our recent paper [7], using the original "forward-backward" (or " \pm " for short) version of the SK formalism. The genus expansion of equilibrium string theory is refined into a sum over triple decompositions of the world sheet topologies. We present our main results in Sec. II, without proofs; the detailed arguments can be found in [7].

In many physical applications, the \pm version of the SK formalism is found to be a little cumbersome [24,25], and it is convenient to perform a redefinition of fields known as the Keldysh rotation. In Sec. III, we extend our analysis from [7] to the Keldysh-rotated formalism. The large- $N$ expansion then predicts an intriguing new structure of the surfaces: Each $\Sigma$ decomposes into a classical and quantum part. We again leave out all proofs; more details can be found in our forthcoming paper [26]. In Sec. IV, we focus our attention on the class of models in which the Feynman ribbon diagrams give a geometric discretization of the metric properties of $\Sigma$, as, e.g., in the old matrix models for noncritical strings. Our refined string perturbation theory then exhibits some additional special features, not necessarily shared by all string theory duals in the more general case.

Nonequilibrium string perturbation theory from large $N$.-How can world sheets be mapped to the target spacetime that incorporates the SK time contour? Figure 1(b) gives some first intuition: When particle
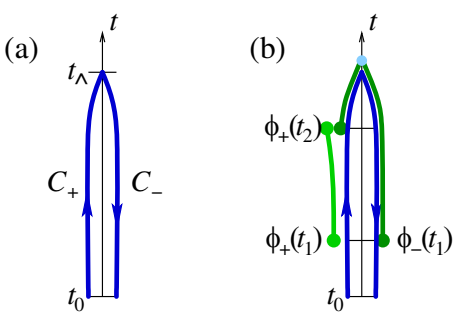

FIG. 1. (a) The Schwinger-Keldysh contour $\mathscr{C}=C_{+} \cup C_{-}$. (b) Worldlines of particles corresponding to two of the four $G_{ \pm \pm}$ propagators: $\left\langle\phi_{+}\left(t_{1}\right) \phi_{+}\left(t_{2}\right)\right\rangle_{0}$ and $\left\langle\phi_{-}\left(t_{1}\right) \phi_{+}\left(t_{2}\right)\right\rangle_{0}$.

worldlines are mapped to the SK contour, the worldlines corresponding to the propagators whose ends are at the two branches of the time contour must cross the point where the two branches meet, and that can be represented graphically by a cut across the propagator. So perhaps when world sheets map to the SK time contour, they should exhibit similar cuts.

In the large- $N$ duality, world sheets are made from ribbon diagrams. Consider the quantum many-body system of Hermitian matrices $M^{a}{ }_{b}(t, \ldots)$, in the adjoint of the symmetry group $\mathrm{SU}(N)$. The ellipsis here refers to all dependence of $M_{ \pm}$on spatial coordinates or additional quantum numbers, which we keep implicit, indicating only the dependence on time. Thus, our results will be universal, regardless of whether $M$ are spacetime fields (such as Yang-Mills gauge fields), relativistic or not, or just matrices in a simple quantum mechanics: Our conclusions will be the same.

We assume that, in equilibrium, the action is of the single-trace form,

$$
S(M)=\frac{1}{g^{2}} \int d t \operatorname{Tr}\left(M^{2}+M^{3}+M^{4}+\cdots\right),
$$

and we study it in the $N \rightarrow \infty$ limit with the "t Hooft coupling $\lambda=g^{2} N$ held fixed. Famously, this expansion leads to a dual perturbative expansion into string world sheet topologies, Eq. (1).

Now we extend this duality away from equilibrium, considering the system of $M$ in the SK formalism. First, we have the usual doubling of fields to $M_{+}(t, \ldots)$ and $M_{-}(t, \ldots)$, representing the values of $M$ at the forward and backward branch of the SK contour. In this forwardbackward formalism, the action that reproduces the diagrammatic rules away from equilibrium can be succinctly written as

$$
S_{\mathrm{SK}}=S\left(M_{+}\right)-S\left(M_{-}\right) \text {, }
$$

although one needs to exercise some care about appropriate conditions where the two parts $C_{ \pm}$of the time contour meet [27]. 
The Feynman rules contain four types of propagators,

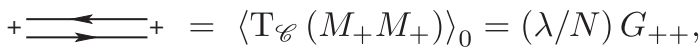

$$
\begin{aligned}
& +\Longrightarrow-=\left\langle\mathrm{T}_{\mathscr{C}}\left(M_{+} M_{-}\right)\right\rangle_{0}=(\lambda / N) G_{+-},
\end{aligned}
$$

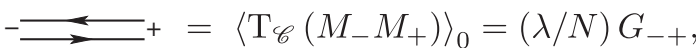

$$
\begin{aligned}
& =-{ }^{-}=\left\langle\mathrm{T}_{\mathscr{C}}\left(M_{-} M_{-}\right)\right\rangle_{0}=(\lambda / N) G_{--},
\end{aligned}
$$

with $T_{\mathscr{C}}$ denoting the time ordering along the contour $\mathscr{C}$. Each vertex gets labeled by a sign,

$$
\begin{array}{ll}
\Rightarrow=-\frac{i N}{\lambda}, & =-\angle=\frac{i N}{\lambda}, \\
+ & =-\frac{i N}{\lambda},
\end{array}
$$

Note that we have also suppressed the $\mathrm{SU}(N)$ indices $a, b, \ldots$ Unlike all the other indices and spatial dependences that we keep implicit and whose role is largely decorative, the $\mathrm{SU}(N)$ indices are of course crucial to our arguments, but they are well represented in the usual way in the graphical form: They are carried by the edges of the ribbon diagrams.

Thus, compared to equilibrium, the novelty of the ribbon Feynman diagrams in nonequilibrium is in the labeling of vertices as + or - . This extra structure, in turn, induces additional topological features on the associated world sheet surfaces. Certainly, there will be portions $\Sigma^{+}$and $\Sigma^{-}$ of $\Sigma$ such that their vertices and propagators reside, respectively, either solely on $C_{+}$or solely on $C_{-}$. These two surfaces should then be joined to form $\Sigma$. The question is, how are they joined? One can begin by indicating each propagator that straddles the two branches of the SK contour (i.e., $G_{+-}$and $G_{-+}$) by putting a cut across it, to indicate that it crosses from the + region to the - region,

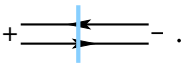

Then one can try to extend these propagator cuts into consistent cuts of the entire world sheets.

A detailed analysis reveals that such cuts cannot be uniquely and usefully extended [7]. More precisely, when extended in a unique way across $\Sigma$, they become complicated graphs on $\Sigma$, not just a collection of a few $S^{1}$ boundaries between $\Sigma^{+}$and $\Sigma^{-}$. To resolve this issue, one needs to widen the cut into a portion of a twodimensional surface with a boundary,

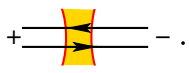

It is these widened cuts that connect smoothly and uniquely across all plaquettes and lead to a decomposition of $\Sigma$ into three topologically meaningful parts characterized by natural topological invariants. In this threefold decomposition, the region of $\Sigma$ that corresponds to the instant $t_{\wedge}$ in time where the two branches of the SK time contour meet is not just a collection of $S^{1}$ boundaries between $\Sigma^{+}$and $\Sigma^{-}$: It is a two-dimensional surface $\Sigma^{\wedge}$, with its own nontrivial topology and its own genus expansion.

Combinatorially, we define this triple topological decomposition of $\Sigma$ as follows. All the + vertices, $G_{++}$ propagators, and all the plaquettes whose all adjacent propagators are $G_{++}$define $\Sigma^{+}$; analogously for $\Sigma^{-}$. Finally, all $G_{+-}$and $G_{-+}$propagators and all the plaquettes with at least one such $G_{ \pm \mp}$ propagator define the wedge region $\Sigma^{\wedge}$. A careful analysis shows [7] that all topologies of the three regions do indeed appear from consistent ribbon diagrams, as long as they respect that their union $\Sigma$ is connected.

Thus, we conclude that, in the nonequilibrium case, the genus expansion (1) of string perturbation theory is refined into a sum over triple decompositions of world sheets,

$$
\mathcal{Z}=\sum_{h=0}^{\infty}\left(\frac{1}{N}\right)^{2 h-2} \sum_{\substack{\text { triple ecompositions } \\ \chi\left(\Sigma^{+} \cup \Sigma^{-} \cup \Sigma^{\wedge}\right)=2-2 h}} \mathcal{F}_{\Sigma^{+}, \Sigma^{-}, \Sigma^{\wedge}}(\lambda, \ldots) .
$$

A typical world sheet that contributes to this sum is shown in Fig. 2.

Keldysh rotation and nonequilibrium string perturbation theory.-The Keldysh rotation is defined by a simple but useful linear change of variables, from the forward and backward fields $M_{ \pm}$to their "classical" and "quantum" counterparts,

$$
M_{\mathrm{cl}}=\frac{1}{2}\left(M_{+}+M_{-}\right), \quad M_{\mathrm{qu}}=\frac{1}{2}\left(M_{+}-M_{-}\right) .
$$

To avoid clutter, we will simply call them $M_{\mathrm{cl}} \equiv M$ and $M_{\text {qu }} \equiv \mathscr{M}$.

In these new variables, the quadratic part of the SK action simplifies and only three propagators are nonzero,

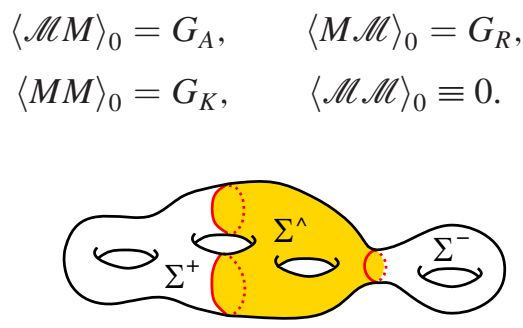

FIG. 2. A typical surface $\Sigma$ and its triple decomposition into the forward region $\Sigma^{+}$, the backward region $\Sigma^{-}$, and the wedge region $\Sigma^{\wedge}$ connecting them. 
Moreover, the information about the dynamics and about the state is now nicely separated: The advanced and retarded propagators $G_{A}$ and $G_{R}$ describe the dynamics, while the entire information about the state is encoded in the "Keldysh propagator" $G_{K}$. This makes the Keldysh rotation both computationally more efficient and physically more intuitive [25,28].

We similarly rotate the sources,

$$
J_{\mathrm{cl}} \equiv J=\frac{1}{2}\left(J_{+}+J_{-}\right), \quad J_{\mathrm{qu}} \equiv \mathscr{J}=\frac{1}{2}\left(J_{+}-J_{-}\right) .
$$

To simplify the construction of Feynman diagrams, we find it useful to introduce a "signpost notation" [26]: Each vertex is equipped with a collection of arrows (i.e., a signpost), one pointing into each quantum end of the vertex. The vertices are
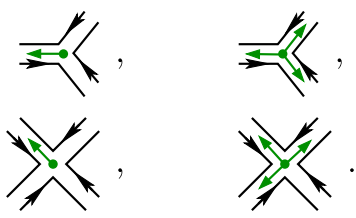

Note that, in full generality, $S_{\mathrm{SK}}$ always gives only vertices with an odd number of $\mathscr{M}$ ends (and hence an odd number of arrows in their signpost). The Feynman rules are such that propagators must match the signposts: Each $\langle\mathscr{M} M\rangle_{0}$ and $\langle M \mathscr{M}\rangle_{0}$ propagator has an arrow pointing into it at its quantum end. Furthermore, if any diagram contains a closed path formed by following the arrows at each vertex, it is zero identically [26].

The Keldysh rotation (10) is a simple transformation of variables, but the two string dual pictures are not expected to be related to each other in any simple way [26]: The relation between the world sheets $\Sigma$ before and after the Keldysh rotation would require a prohibitively complicated resummation of many ribbon diagrams. Thus, we find two distinct dual string expansions, each with its unique features.

In the Keldysh-rotated formalism for the large- $N$ expansion, we find that the sum over surface topologies is again refined, but in a different way [26]: Each $\Sigma$ is decomposed into its classical part $\Sigma^{\mathrm{cl}}$ and quantum part $\Sigma^{\mathrm{qu}^{\mathrm{u}}}$. Combinatorially, these parts are defined as follows: All $G_{A}$ and $G_{R}$ propagators, all internal vertices, and all the plaquettes without adjacent $G_{K}$ propagators define the classical part $\Sigma^{\mathrm{cl}}$. All the $G_{K}$ propagators and all the plaquettes with at least one adjacent $G_{K}$ propagator comprise the quantum part $\Sigma^{q u}$. Note that the nice separation of the dynamics from the many-body state of the large- $N$ system extends to the string side: The information about the state resides in $G_{K}$ and is now entirely carried by $\Sigma^{\text {qu }}$. In contrast, $\Sigma^{\mathrm{cl}}$ contains all the elements that only know about the dynamics.
Further analysis shows [26] that all possible topologies of $\Sigma^{\mathrm{cl}}$ and $\Sigma^{\mathrm{qu}}$ emerge from consistent ribbon diagrams, as long as they give a connected $\Sigma$. We conclude that in the Keldysh-rotated description, the sum over topologies in nonequilibrium string perturbation theory is refined to a sum over the world sheet decompositions into their classical and quantum parts,

$$
\mathcal{Z}(J, \mathscr{J})=\sum_{h=0}^{\infty}\left(\frac{1}{N}\right)^{2 h-2} \sum_{\substack{\text { double decompositions } \\ \chi\left(\Sigma^{\mathrm{cl}}\right)+\chi\left(\Sigma^{\mathrm{qu}}\right)=2-2 h}} \mathcal{F}_{\Sigma^{\mathrm{cl}}, \Sigma^{\mathrm{qu}}}(\lambda ; J, \mathcal{J}) .
$$

We have added the dependence on the sources because all the individual vacuum diagrams can be shown to be identically zero [26]. This equation then holds for all the correlation functions of $M$ and $\mathscr{M}$. A typical example of a surface contributing to the sum is shown in Fig. 3.

Random triangulations. - The specific coefficients $\mathcal{F}$ in our triple- and double-decomposition sums encode all the world sheet dynamics and are therefore generally out of reach for our universal analysis of the string loop expansion.

For special subclasses of models, however, we can obtain further insights without losing the universal nature of our conclusions. Consider the subclass consisting of those large- $N$ systems, for which the ribbon diagrams serve as a tool to discretize the path-integral sum over world sheet geometries and to regulate the Einstein-Hilbert action with a cosmological constant (coupled perhaps to simple types of world sheet matter). A typical example is given by the "old-fashioned" matrix models of nonperturbative noncritical string theories in $d \leq 1+1$ spacetime dimensions (see [29-31] for reviews). Even though much has been learned about the matrix models from the more modern perspective of D-branes and tachyon condensation [32,33] (see also [29]), the original reasoning that uses ribbon diagrams to define the world sheet path integral as a sum over random triangulations is still perfectly valid. In this construction, each Feynman ribbon diagram is viewed as a dual to a triangulation (or more generally, a simplicial decomposition) of $\Sigma$. One unit $A$ of discretized area is assigned to each vertex of the large- $N$ ribbon diagram, i.e., to each plaquette of the dual diagram. The regulated world sheet path integral is then given by the sum over all Feynman diagrams, and its continuum limit is described by a certain double scaling limit of the large- $N$ theory.

With this reminder, we are ready to identify additional universal features of the topological genus expansion of

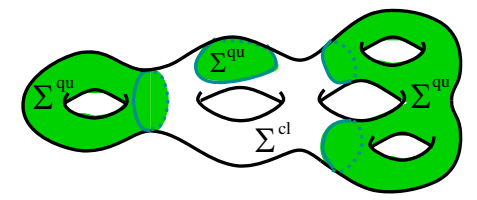

FIG. 3. A typical surface $\Sigma$ and its decomposition into the classical and quantum part $\Sigma^{\mathrm{cl}}$ and $\Sigma^{\mathrm{qu}}$. 
nonequilibrium string theory in this special class of models. Recall how we assigned the various combinatorial elements of the ribbon diagrams in the \pm formalism to the three parts of the triple decomposition of $\Sigma$ : All vertices were naturally assigned to $\Sigma^{+}$and $\Sigma^{-}$, with the wedge region $\Sigma^{\wedge}$ consisting only of certain propagators and plaquettes. When one unit $A$ of the geometric area is ascribed to each vertex, this implies that-despite being topologically two dimensional — the wedge region $\Sigma^{\wedge}$ of the world sheet has zero regulated area. It is likely that this feature should persist also in the continuum limit.

The same is true in the Keldysh-rotated formalism about the quantum part $\Sigma^{\mathrm{qu}}$ of the world sheet: Only certain plaquettes and propagators were assigned to $\Sigma^{q u}$ in our combinatorial construction, with all vertices belonging to $\Sigma^{\mathrm{cl}}$. Thus, $\Sigma^{\mathrm{qu}}$ carries zero regulated total area. We reach a fairly universal conclusion, valid for the special broad class of random triangulations models: In the dual string theory, $\Sigma^{\wedge}$ and $\Sigma^{\mathrm{qu}}$ are topologically two dimensional and carry their own genus expansion, yet geometrically they effectively appear to be at most one-dimensional objects (unless they overcome this tendency by acquiring large anomalous dimensions in the continuum limit).

Conclusions. - In two distinct formulations of the SK formalism for the large- $N$ expansion of nonequilibrium systems with matrix degrees of freedom, we found that each leads to a distinct refinement of the sum over world sheet topologies in string perturbation theory. New topological invariants are now available (such as the Euler numbers of $\Sigma^{+}, \Sigma^{-}$, and $\Sigma^{\wedge}$ in the original \pm formalism or of $\Sigma^{\mathrm{cl}}$ and $\Sigma^{\mathrm{qu}}$ in the Keldysh-rotated formalism), and it is natural to expect that they can be weighted by different values of the string coupling $g_{+}, g_{-}$, and $g_{\wedge}$ ( or $g_{\mathrm{cl}}$ and $g_{\mathrm{qu}}$ ). Indeed, we envision a situation much like in equilibrium, where in some solutions, the string coupling $g_{s}$ can be spacetime dependent. In the SK context, a time-dependent $g_{s}$ could lead to different effective values of the string coupling in the corresponding regions of the SK time contour.

In order to gain further insights into such open questions, it is important to look at specific dynamical examples of string theory, where some aspects of the world sheet dynamics can be controlled from first principles, and the predicted structure of nonequilibrium string perturbation theory can be directly verified. A particularly natural candidate would be the maximally supersymmetric AdS/CFT correspondence: Revisiting its origin from coincident D-brane systems, now taken out of equilibrium, could provide a bridge between the relatively well-understood spacetime picture [14-19] and the sought-for world sheet description of the Schwinger-Keldysh formalism for nonequilibrium systems.

This work has been supported by NSF Grants No. PHY1820912 and No. PHY-1521446.
[1] J. Zaanen, Y.-W. Sun, Y. Liu, and K. Schalm, Holographic Duality in Condensed Matter Physics (Cambridge University Press, Cambridge, England, 2015).

[2] S. A. Hartnoll, A. Lucas, and S. Sachdev, arXiv:1612.07324.

[3] P. Hořava, Phys. Rev. Lett. 95, 016405 (2005).

[4] The Birth of String Theory, edited by A. Cappelli, E. Castellani, F. Colomo, and P. Di Vecchia (Cambridge University Press, Cambridge, England, 2012).

[5] J. S. Schwinger, J. Math. Phys. (N.Y.) 2, 407 (1961).

[6] L. Keldysh, Zh. Eksp. Teor. Fiz. 47, 1515 (1964).

[7] P. Hořava and C. J. Mogni, arXiv:2008.11685.

[8] P. Hájiček, in The Second Marcel Grossmann Meeting on the Recent Developments of General Relativity (North Holland, Amsterdam, 1979), p. 483.

[9] R. D. Jordan, Phys. Rev. D 33, 444 (1986).

[10] S. Weinberg, Phys. Rev. D 72, 043514 (2005).

[11] S. Weinberg, Phys. Rev. D 74, 023508 (2006).

[12] S. Weinberg, Phys. Rev. D 77, 123541 (2008).

[13] D. Baumann and L. McAllister, Inflation and String Theory (Cambridge University Press, Cambridge, England, 2015).

[14] K. Skenderis and B. C. van Rees, Phys. Rev. Lett. 101, 081601 (2008).

[15] K. Skenderis and B. C. van Rees, J. High Energy Phys. 05 (2009) 085.

[16] J. de Boer, M. P. Heller, and N. Pinzani-Fokeeva, J. High Energy Phys. 05 (2019) 188.

[17] F. M. Haehl, R. Loganayagam, and M. Rangamani, J. High Energy Phys. 06 (2017) 069.

[18] F. M. Haehl, R. Loganayagam, and M. Rangamani, J. High Energy Phys. 06 (2017) 070.

[19] C. P. Herzog and D. T. Son, J. High Energy Phys. 03 (2003) 046.

[20] G. 't Hooft, Nucl. Phys. B72, 461 (1974).

[21] G. 't Hooft, Nucl. Phys. B75, 461 (1974).

[22] A. M. Polyakov, in Proceedings of the Les Houches Summer School on Gravitation and Quantizations, Session LVII (North Holland, Amsterdam, 1993), p. 783.

[23] J. M. Maldacena, Adv. Theor. Math. Phys. 2, 231 (1998).

[24] G. Vilkovisky, Lect. Notes Phys. 737, 729 (2008).

[25] J. Rammer, Quantum Field Theory of Non-Equilibrium States (Cambridge University Press, Cambridge, England, 2007).

[26] P. Hořava and C. J. Mogni, arXiv:2010.10671.

[27] A. Kamenev, Field Theory of Non-Equilibrium Systems (Cambridge University Press, Cambridge, England, 2011).

[28] G. Stefanucci and R. van Leeuwen, Nonequilibrium ManyBody Theory of Quantum Systems (Cambridge University Press, Cambridge, England, 2013).

[29] Y. Nakayama, Int. J. Mod. Phys. A 19, 2771 (2004).

[30] I. R. Klebanov, in Spring School on String Theory and Quantum Gravity (World Scientific, Singapore, 1991), p. 30.

[31] P. H. Ginsparg and G. W. Moore, in Theoretical Advanced Study Institute (TASI 92): From Black Holes and Strings to Particles (World Scientific, Singapore, 1993), p. 277.

[32] M. R. Douglas, I. R. Klebanov, D. Kutasov, J. M. Maldacena, E. J. Martinec, and N. Seiberg, arXiv:hep-th/ 0307195.

[33] J. McGreevy and H. L. Verlinde, J. High Energy Phys. 12 (2003) 054. 